\title{
BMJ Open Proposing evidence-based strategies to strengthen implementation of healthcare reform in resource-limited settings: a summative analysis
}

\author{
Tsegahun Manyazewal, ${ }^{1,2}$ Martha J Oosthuizen, ${ }^{1}$ Mokgadi C Matlakala ${ }^{1}$
}

To cite: Manyazewal T, Oosthuizen MJ, Matlakala MC. Proposing evidence-based strategies to strengthen implementation of healthcare reform in resource-limited settings: a summative analysis. BMJ Open 2016;6:e012582. doi:10.1136/bmjopen-2016012582

- Prepublication history and additional material is available. To view please visit the journal (http://dx.doi.org/ 10.1136/bmjopen-2016012582).

Received 10 May 2016 Revised 15 August 2016 Accepted 30 August 2016

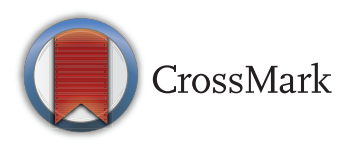

${ }^{1}$ Department of Health Studies, College of Human Science, University of South Africa, Pretoria, South Africa ${ }^{2}$ Ethiopian Public Health Association, Addis Ababa, Ethiopia

Correspondence to Dr Tsegahun Manyazewal; tsegahunm@gmail.com

\section{ABSTRACT}

Objectives: Many resource-limited countries have adopted and implemented healthcare reform to improve the quality of healthcare, but few have had much impact and strategies in support of these efforts remain limited. We aimed to explore and propose evidence-based strategies to strengthen implementation of healthcare reform in resource-limited settings.

Design: Descriptive and exploratory designs in two phases. Phase I involved assessing the effectiveness of the healthcare reform implemented in Ethiopia in the form of business process reengineering, with evidence compiled from healthcare professionals through a selfadministered questionnaire; and phase II involved proposing strategies and seeking consensus from experts using Delphi method.

Setting: Public hospitals in central Ethiopia.

Participants: 406 healthcare professionals and 10 senior health policy experts.

Findings: The healthcare reform that we evaluated was able to restructure hospital departments into case teams, with the goal of adopting a 'one-stop shopping' approach. However, shortages of critical infrastructure, furniture and supplies and job dissatisfaction continued to hamper the system. The most important predictors that influenced implementation of the reform were financial resources, top management commitment and support, collaborative working environment and information technology (IT). Five strategies with 14 operational objectives and 67 potential interventions that could strengthen the reform are proposed based on their strategic priority, which are as follows: reinforce patient-centred quality of care services; foster a healthy and respectful workforce environment; efficient and accountable leadership and governance; efficient use of hospital financing and maximise innovations and the use of health technologies.

Conclusions: Effective implementation of healthcare reform remained a challenge for governments in resource-limited settings. Resilient operational, clinical and governance functions of health systems, as well as a motivated and committed health workforce, are important to move healthcare reform processes forward. Political commitments at this juncture might be critical though there need to be a clear demarcation between political and technical engagements.

\section{Strengths and limitations of this study}

- The study included all public hospitals of Addis Ababa, Ethiopia, which have been serving citizens in multiple disease prevention, care and treatment services and dedicated as training and academic centres of excellence for decades.

- The study involved senior health policy experts in the development of the strategies.

- It was well intentioned that the study included all healthcare professionals without any discrimination done among professional disciplines.

- The study collected the maximum possible sample size and conducted the study in a very professional manner.

- The study did not solicit for the views of administrative staff and we think their inputs may have further strengthened the study findings, while the possible effect of this has been mitigated by inclusion of healthcare providers who also have administrative role.

\section{BACKGROUND}

Irrespective of their limited resources, many developing countries have initiated healthcare reform to provide broad access to standard healthcare while simultaneously cutting down costs. ${ }^{1}$ Given the scope of healthcare reforms, these countries' decisions are often political processes which lie with governments and their own unique health concerns. $^{2}$ In these settings, civil service institutions such as public health facilities fall under the influence of politics to impose law and order to implement government policies and strategies. ${ }^{3}$ These countries have been attempting to reform their healthcare services using different management models, but copied from the well-developed countries, often out of context and without considering their resource potentials and management capacity. ${ }^{4}{ }^{5}$ Business Process Re-engineering (BPR) is among the major management approaches that have been 
adapted and implemented by developing countries with the goal of improving healthcare services' operational settings and strengthening the way healthcare is delivered. ${ }^{6}$

Closely allied with the healthcare reform in these settings is its implementation technique such as BPR. Studies indicate that market economic principles are good for generating wealth but poor at improving health and social welfare, ${ }^{78}$ and looking at healthcare environments as market places were reported to radically affect health professions and professionals. ${ }^{8}$ For instance, healthcare reform, when implemented using BPR as a tool, was shown to be swayed by the commitment and support of high-level management, ${ }^{9-11}$ financial resources, ${ }^{12-14}$ training demands ${ }^{15}$ and management structure. ${ }^{16-18}$

The passage of healthcare reform needs to thrive to come up with solutions to both patients and the health workforce. Technical competency of the health workforce who ultimately implement the new healthcare reform process have an imperative role in the success or failure of the reform. ${ }^{15}{ }^{19-21}$ If the health workforces are absent in the reform process, they may feel they will be displaced from their job position due to the redesigned process or may feel job dissatisfaction which can also result in poorer patient outcomes, financial losses and failure of the reform. ${ }^{22} 23$

Different African countries such as Ethiopia, Kenya, Uganda, Tanzania, South Africa and Lesotho have been struggling to reform their healthcare and achieve the vision of the African Health Strategy 2007-2015 to an integrated and prosperous Africa, free of the heavy burden of disease, disability and premature death. ${ }^{24}$ The healthcare system of Ethiopia is part of the larger healthcare system in resource-limited settings, a public sector-reliant healthcare service with limited human and financial resources. In the wave of the pressure to improve healthcare services' operational settings and strengthen the delivery of healthcare, the Ethiopian government urged a countrywide healthcare reform initiative in the form of BPR. The reform has been implemented across all public healthcare sectors through a series of training sessions for managers and service providers at all levels followed by changes in staff deployment, specific job assignments and the recruitment of new staff. However, despite implementation of the reform, the determinants of success or failure were poorly documented. In general, we found out from the review of relevant literature ${ }^{25-30}$ that there were certain study reports which evaluated the effectiveness of healthcare reforms on the quality of healthcare system in resourcelimited settings. However, beyond proposing recommendations, none was able to propose evidence-based strategies in support of the implementation of the reforms in these settings.

Thus, the aim of this study is to explore and propose evidence-based strategies to strengthen implementation of healthcare reform in resource-limited settings. To attain this aim, we formulated three research questions as follows: What are the perceived effects of healthcare reform on the overall healthcare performance? What factors influence implementation of healthcare reform? What strategies are required to strengthen implementation of healthcare reform?

\section{METHODS}

A two-phase summative evaluation and analysis was carried out in Ethiopia between January and June 2015. Phase I involved evaluating the effectiveness of the healthcare reform implemented in Ethiopia in the form of BPR. A descriptive study was conducted in the form of survey to gather quantitative data from healthcare professionals through a self-administered questionnaire. Addis Ababa was selected among the 11 administrative divisions of Ethiopia considering its presence as the largest city and capital of Ethiopia and the political capital of Africa where the headquarters of the African Union, United Nations Economic Commission for Africa and numerous other continental and international organisations are based. It is highly resourced with hospitals that provide advanced preventive and curative healthcare services. The Addis Ababa Health Bureau administers six $(n=6)$ public hospitals which are anticipated to deliver advanced preventive and curative health services, from which all that have been implementing the BPR healthcare reform since its inception in 2008-2009 $(n=5)$ were purposively selected as study sites. As the reform was implemented only in public health sectors, private sectors were not considered in this study.

The study respondents were sourced from all full-time healthcare providers that were working in the study sites at the time of data collection $(n=1681)$. Of these, all who were hired at least 1 year before the inception of the reform $(n=476,28 \%)$ were drawn with purposive sampling technique to select respondents who knew the performances of the study sites before implementation of the reform and who could better analyse the changes that occurred due to the reform. The healthcare providers included medical doctors, laboratory professionals, nurses, health officers, pharmacists, dentists and sanitarians.

A self-designed, structured questionnaire was prepared in a close-ended 5-level Likert scale ${ }^{31}$ based on the 'health system performance' framework ${ }^{32}$ which was used as the driving theoretical grounding of the study. The framework presents indicators for five key dimensions of health system performance, namely, quality, access, equity, efficiency and sustainability, which maps the linkages between healthcare reform, changes in health system performance and changes in health status. ${ }^{31}$ The approach provides a rationale for focusing on system performance as one of the principal ways to evaluate the results of healthcare reform.

Accordingly, the questionnaire was designed to describe the perceived effect of the BPR healthcare 
reform on quality, access, equity, efficiency and sustainability of healthcare services. In the 'quality' component, investigation has been made with 32 items (Cronbach's $\alpha=0.960)$ to analyse the reform's effect on meeting the intended quality of health services. The quality investigation was guided by three healthcare quality dimensions: structure-process-outcome, as proposed in the Donabedian quality-of-care framework. ${ }^{33}$ The quality 'outcome' had 22 items (Cronbach's $\alpha=0.958$ ): eight items focusing on patient-provider interaction, four items on documentation and monitoring and evaluation, and nine items on provider-management interactions constructed from the targets the Ethiopian government had depicted in its reform document. ${ }^{34}$ The quality 'process' part had eight items (Cronbach's $\alpha=0.921$ ) aimed at determining the appropriateness of the methods and procedures followed in the implementation of the reform. 'Structure' had two items aimed at determining improvements of the overall structure of the hospitals to meet the daily workflow.

Improvement in 'access' was determined by including 25 items (Cronbach's $\alpha=0.960$ ) in the questionnaire. This was made based on the five dimensions of healthcare access: physical, economic, temporal, cultural and approachability dimensions. ${ }^{35-37}$ The 'physical' dimension determined the availability of enough work space, furniture, equipment, supplies, medications, reagents, communication materials and other supplies in the hospitals after implementation of the reform. The 'economic' dimension determined the overall effect of the reform on financing and financial management system, and 'temporal' dimension determined the effect of the reform on improving turn-around-time of the hospitals' healthcare services. 'Cultural' dimension evaluated the acceptability of the hospitals' services, and 'approachability' dimension evaluated the effect of the reform on improving awareness of the community that some form of health services exists, can be reached and have an impact on their health.

The 'equity' section determined the availability of adequate resources and systems in the hospitals that would fairly benefit every citizen by adding four items in the questionnaire. 'Efficiency' component determined the technical, economic and allocative processes related to how and which services are produced in the reform process using 12 items. While 'sustainability' of hospitals' services to continue functioning and initiate changes so as to continuously improve performance was determined through including nine relevant items in the questionnaire.

Respondents received and completed the study questionnaire on paper-based form while they were on their working area. Each of the five responses in the questionnaire had a numerical value (1-5), in which the highest two scoring answers ( 4 and 5 ) were perceived as positive response answers, the lowest two scoring answers (1 and 2) were considered negative response answers, and the middle response answer (3) was perceived neutral. As the question's items were grouped into healthcare performance dimensions, a scale score was computed as the mean of the scales' item scores.

On the other side, an in-depth review of literature was carried out to identify critical factors that could influence the success of BPR programmes. As a result, six BPR critical success factors were identified, which were as follows: financial resources, top management commitment and support, training, collaborative working environment, flatter structure and IT. ${ }^{12} 1620212238$ These factors have been used as a guide in identifying and analysing the factors that influence implementation of the BPR healthcare reform. From the study's questionnaire, the item stating 'the hospital becomes a better treatment facility' was taken as the outcome variable to indicate whether or not there was hospital service improvement. This item had original responses classified in Likert scale. The responses were dichotomised into 'Good' or 'Poor' answers by taking the 'Strongly agree' and 'Agree' responses as a 'Good' value while 'Strongly disagree', 'Disagree' and 'Neutral' as 'Poor' value for feasibility of analysis and interpretation. While the six BPR critical success factors were taken as the explanatory variables. For each of the six success factors, three items which best describe the factor were pooled from the questionnaire and the responses given to the items by the respondents analysed as a cumulative effect. Responses were taken as 'Good' value if at least two of the three items had a 'Strongly agree' or 'Agree' response in the original Likert scale questionnaire, while the remaining responses were taken as 'Poor' value. The six critical success factors and the items included under each factor are summarised in online supplementary appendix 1 . Associations of health service improvement with the six explanatory variables were tested independently using bivariate analysis. Based on the result, all the independent variables were selected for the logistic regression analysis. Subsequently, multivariate logistic regression analysis has been conducted to exclude confounders.

Phase II of the study involved proposing strategies and seeking consensus from experts using Delphi method ${ }^{39}$ to arrive at consensus on the proposed strategies. In-depth analysis was conducted on responses from phase I to explore and propose strategic priorities that are likely to strengthen implementation of the BPR healthcare reform and attract significant impact on future healthcare systems in resource-limited settings. The major gaps were categorised into seven thematic areas. Ten $(n=10)$ senior health policy experts were purposively drawn through personal networks and referral by other experts from government institutions, development partners, universities, non-governmental organisations, healthcare administrators and professional associations that were working closely with Ethiopian Federal Ministry of Health $(\mathrm{FMoH})$. Inclusion criteria were holding a $\mathrm{PhD}$ in the field of health policy or public health, 15-20 years of extensive services in national and/or international health programmes, possessing a wide experience of lecturing 
in universities and conducting researches focusing on health policies, and knowledgeable in health policies. The inclusion criteria were formulated to focus only on vastly superior expertise in the field, though there had been senior health policy expertise in the country that may not fulfil one or more of the criteria but have the required experience and knowledge of healthcare systems in resource-limited settings. In the first Delphi technique, a questionnaire which consisted of a set of seven strategic priorities was developed and the experts ordered the strategies by priority of importance and considered how likely it is that certain changes will occur in the years 2016-2020 due to each strategic priority. In the second round, another questionnaire that aimed at informing the panel experts about findings of the first round of the study and seeking further consensus about two strategic priorities that had a similar score on the first Delphi study was developed and shared for the experts to respond. Based on the experts' response, of the seven strategic priorities, five were selected for further analysis for having above 2 (-) SD score. The five strategic priorities were further explored and discussed with rationale, operational objectives and potential interventions to use to enrich the quality-of-care interventions in resourcelimited settings through continuous review, refinement and adjustment of the reform as required.

Written informed consents were developed for each respondent to read and sign before moving on to filling the questionnaires. There was no compensation or reimbursement to the respondents and this had been communicated to respondents in advance. In both phases, data were exported into IBM SPSS V.20 software and analysed. Cronbach's $\alpha$ test, ${ }^{40}$ descriptive statistics, $\chi^{2}$, logistic regression analysis, ${ }^{40}$ principal component analysis, ${ }^{41}$ weighted median score, ${ }^{41}$ Mann-Whitney U test and Kruskal-Wallis test, ${ }^{42}$ and adjusted and standard satisfaction scores ${ }^{43}$ were conducted for data analysis.

\section{RESULTS}

A total of 410 healthcare professionals completed the survey representing an $88 \%$ response rate, where data from 406 were fully completed and presented for analysis. Majority of the respondents $(84.2 \%)$ had at least 1st degree and most of them $(74.9 \%)$ were nurses followed by medical doctors $(8.6 \%)$ and medical laboratory professionals $(5.9 \%)$. For work experience, $49.8 \%$ and $0.7 \%$ of respondents had work experience as health professional for 10-19 and 30-39 years, respectively. As per the inclusion criteria, all of them were hired in the hospitals at least a year ahead of the inception of the reform.

For 'quality' of healthcare services, descriptive analysis of the weighted median score for $52 \%$ of respondents indicated that implementation of the BPR healthcare reform did not improve the quality of healthcare services. Forty-two per cent indicated that the reform did not meet the perceived patients-provider interaction.
The time-limit $(10 \mathrm{~min})$ allotted in the reform document for patients getting beds has not been met $(84 \%)$, while there was a relatively positive concurrence $(58 \%)$ that the reform enabled patients to obtain their medical certificate within an hour as specified in the reform document. Only $25.1 \%$ respondents agreed that the reform allowed reporting systems of the hospitals to be easy and time-efficient and only 32\% agreed that hospital guidelines and protocols are up-to-date and appropriate. The respondents' feedback showed that the hospital staff were not promoted to a relatively higher position $(60.6 \%)$ nor got recognition of their outstanding performance $(62.8 \%)$ due to the reform. Besides, the reform did not allow for increases in salary of staff $(63.1 \%)$ nor created a better feeling of overall job satisfaction among staff $(56.2 \%)$, while it created a relatively better working relationship among staff $(50.2 \%)$. The overall quantitative analysis of findings indicated that provider-management interaction of the hospitals is still weak.

According to the weighted median score, $52 \%$ of respondents indicated that appropriate procedures have not been followed in the processing of the BPR healthcare reform. Among the major gaps in the reform implementation process was training. About $60.6 \%$ of the respondents claimed that adequate training had not been provided to all staff throughout the reform implementation process. Regarding the composition of the reform team, $40.9 \%$ of respondents argued that the right team members had not been assigned to process the reform and $49.8 \%$ claimed that feedbacks from patients and data from pilot tests were not incorporated to the reform. Similarly, $52.5 \%$ of respondents argued that there is no improvement in the structure of the hospitals.

For 'access', $50 \%$ of the healthcare providers reported that the reform was not effective to satisfy the perceived health service access and $50.7 \%$ of respondents indicated that the reform enabled the hospitals organised with case teams that have well-defined rooms or spaces adequate for the daily workflow. Yet, according to the respondents, there were other physical barriers which were compromising their day-to-day activities. For instance, the respondents claimed that after implementation of the reform, the hospitals still lack to have enough office furniture $(55.9 \%)$, stationery materials $(58.6 \%)$ and reagents and drugs (53\%). Implementation of the reform did not empower the hospitals to get equipped with internet services $(81.8 \%)$, functioning computers $(56 \%)$ and a functioning landline telephone to call within and outside the hospital $(58.9 \%)$. Preventive and curative maintenance of diagnostic equipment were also described as ineffective. The overall finding indicated that the reform was unable to address the shortages or absence of critical furniture, supplies and infrastructure that are highly needed for the hospitals' day-to-day services. About $34.7 \%$ respondents agreed that efficient and effective healthcare 
financing systems are in place after implementation of the reform, while $20 \%$ disagree and the majority $(45.3 \%)$ were neutral. Yet, $40.6 \%$ respondents claimed that corruption suspects in the hospitals still exist. In general, $37 \%$ responses agreed that there were improvements in economic dimensions of the hospitals, $27 \%$ disagree and $36 \%$ were neutral. While the reform reasonably addressed temporal dimensions of healthcare reform performance measures, with $54 \%$ level of agreement out of the total responses. Regarding cultural dimension of access, $55.2 \%$ concurred that the reform enabled patients receive healthcare services using a mode of communications suitable to them. On the other side, the majority of respondents $(83.3 \%)$ agreed that there is no patient discrimination in the hospitals since the reform has been implemented, and this was the highest ever recorded score in the study. The overall analysis revealed that the reform was reasonably capable of addressing the cultural dimensions of healthcare reform performance, with only $0.6 \%$ level of disagreement out of the total number of responses recorded.

For 'equity', based on analysis of weighted median score, $61 \%$ of respondents agree that healthcare equity has been improved due to implementation of the reform. Results indicated that the hospitals are giving medical services with reasonable prices, including free services for patients who cannot afford it. Above all, $70 \%$ of respondents indicated that patients with different socioeconomic, demographic, ethnic and/or gender groups have equal access to the hospitals' services. For 'efficiency', according to the weighted median score, $51 \%$ of respondents concurred that the reform did not improve efficiency of healthcare services. Forty-three per cent of respondents agreed that the best use of economic resources has been achieved in the reform implementation process, and $44.6 \%$ of respondents agree that enough and competent healthcare workers were in place in the reform implementation process. However, $44 \%$ of respondents argued that the role and capacity of the hospitals' high-level management in the reform implementation process was insufficient. For 'sustainability', $52.7 \%$ of respondents claimed that the reform did not improve sustainability of the public hospitals.

The most important predictors that influenced implementation of the reform were financial resources $(\mathrm{AOR}=3.54,95 \%$ CI 1.97 to 6.33$)$, top management commitment and support ( $\mathrm{AOR}=2.27,95 \%$ CI 1.15 to $4.47)$, collaborative working environment $(\mathrm{AOR}=1.77$, $95 \%$ CI 1.00 to 3.11 ) and IT (AOR=3.15, 95\% CI 1.57 to 6.32) (table 1).

\section{PROPOSED STRATEGIES}

The major gaps identified in phase I of the study were clustered into seven thematic areas, which were as follows: patient, provider, leadership, finance, audit, reform and technology. With these, seven strategic priorities were formulated which were likely to attract significant impact on future healthcare systems. The seven strategic priorities were as follows:

Reinforce patient-centred quality of care services: This strategic priority focused on patients and emanated mainly from the gaps identified on patient-provider interaction, the temporal, cultural and approachability dimensions of healthcare access, and the equity of healthcare services.

Foster a healthy and respectful workforce environment: This strategic priority focuses on healthcare providers and emanated from the major gaps identified while the relationship between the BPR healthcare reform and healthcare providers' job satisfaction and interaction with hospital management was examined. The in-depth analysis made on continuing education, salary and benefits, management style, tasks, work environment, workload, moral satisfaction and job stability of the health workforce flagged up the prompt need for a healthy and respectful workforce environment in the public healthcare sectors.

Efficient use of hospital financing: This strategic priority was emanated from findings in phase I which identified limitation in financial resources and poor healthcare financing system as the major influencing factor in the implementation of the healthcare reform.

Initiate new healthcare reform: This strategic priority was proposed from findings in phase I which witnessed loosen competency of the reform to ensuring sustainability of the hospitals' services and the inappropriate processes followed in the implementation of the reform.

Ensure efficient and accountable leadership and governance: This strategic priority was emanated from the leadership and governance gaps identified while the current healthcare delivery performance of public hospitals was evaluated. The findings indicate the need for efficient and accountable leadership and governance practices to make the healthcare system effective enough.

Maximise innovations and the use of health technologies: This strategic priority was rooted in findings which identified critical gaps in the development and usage of IT, medical products/technologies, and gaps in the meaningful exchange of information.

Trigger health system audits: This strategic priority was proposed based on the overall findings of phase I of the study which indicated the lack of a well-functioning monitoring and evaluation system across the administrative hierarchies of the public health sector.

Based on findings of the two rounds of Delphi technique conducted (table 2), five of the seven strategic priorities were selected and developed well with 14 operational objectives and 67 potential interventions.

The strategies, based on their priorities, were as follows: (1) reinforce patient-centred quality of care services; (2) foster a healthy and respectful workforce environment; (3) efficient and accountable leadership and governance; (4) efficient use of hospital financing and (5) maximise innovations and the use of health technologies. Each strategic priority consists of the rationale based on findings from phase I of the study, strategic approach and the key interventions needed. The 
Table 1 Logistic regression analyses of the relative effect of BPR critical success factors on health service improvement

\begin{tabular}{|c|c|c|c|c|c|c|}
\hline \multirow[b]{3}{*}{ Factors } & \multicolumn{5}{|c|}{ Healthcare services } & \multirow[b]{3}{*}{ Adjusted OR (95\% Cl) } \\
\hline & \multicolumn{2}{|l|}{ Frequency } & \multirow[b]{2}{*}{ df } & \multirow[b]{2}{*}{ Significance } & \multirow[b]{2}{*}{ Crude OR $(95 \% \mathrm{CI})$} & \\
\hline & Improved & Not improved & & & & \\
\hline \multicolumn{7}{|c|}{ Adequate financial resources } \\
\hline Poor & 145 & 35 & \multirow[t]{2}{*}{1} & \multirow[t]{2}{*}{$0.000^{*}$} & $1^{\mathrm{R}}$ & \multirow[t]{2}{*}{3.54 (1.97 to 6.33$)$} \\
\hline Good & 59 & 167 & & & 11.72 (7.30 to 18.83$)$ & \\
\hline \multicolumn{7}{|c|}{ Top management commitment and support } \\
\hline Poor & 162 & 49 & \multirow[t]{2}{*}{1} & \multirow[t]{2}{*}{$0.018^{*}$} & $1^{R}$ & \multirow[t]{2}{*}{2.27 (1.15 to 4.47$)$} \\
\hline Good & 42 & 153 & & & 12.04 (7.55 to 19.22$)$ & \\
\hline \multicolumn{7}{|c|}{ Collaborative working environment } \\
\hline Poor & 155 & 72 & \multirow[t]{2}{*}{1} & \multirow[t]{2}{*}{$0.050^{*}$} & $1^{R}$ & \multirow{2}{*}{$1.77(1.00$ to 3.11$)$} \\
\hline Good & 49 & 130 & & & 5.71 (3.71 to 8.79$)$ & \\
\hline \multicolumn{7}{|c|}{ Flatter structure } \\
\hline Poor & 169 & 63 & \multirow{2}{*}{1} & \multirow[t]{2}{*}{0.092} & $1^{\mathrm{R}}$ & \multirow{2}{*}{$1.80(0.91$ to 3.55$)$} \\
\hline Good & 35 & 139 & & & 10.65 (6.66 to 17.05$)$ & \\
\hline \multicolumn{7}{|c|}{ Information technology } \\
\hline Poor & 187 & 90 & \multirow[t]{2}{*}{1} & \multirow[t]{2}{*}{$0.001^{*}$} & $1^{\mathrm{R}}$ & \multirow[t]{2}{*}{3.15 (1.57 to 6.32$)$} \\
\hline Good & 17 & 112 & & & 13.70 (7.75 to 24.18$)$ & \\
\hline \multicolumn{7}{|l|}{ Training } \\
\hline Poor & 175 & 73 & \multirow[t]{2}{*}{1} & \multirow[t]{2}{*}{0.218} & $1^{\mathrm{R}}$ & \multirow[t]{2}{*}{$\dagger$} \\
\hline Good & 29 & 129 & & & 10.66 (6.56 to 17.35$)$ & \\
\hline
\end{tabular}

strategies considered what affordable approach would lead public hospitals to the most success, what kind of facility the hospitals want to become and what businesses the hospitals are. The strategies developed align with the draft Health Sector Transformation Plan (HSTP) developed by the FMoH for the years $2016-2020,{ }^{44}$ the core components of the BPR healthcare reform, and the objectives, purposes and goals of public hospitals. The strategic priorities are as follows.

\section{Strategic priority l: reinforce patient-centred} quality-of-care services

Rationale

The current quality of service provided by public hospitals that undergo healthcare reform have serious challenges in

Table 2 Ranking of strategic priorities by panel experts

\begin{tabular}{lll}
\hline Strategic priority & $\begin{array}{l}\text { Mean } \\
\text { score }\end{array}$ & Rank \\
\hline $\begin{array}{l}\text { Reinforce patient-centred quality of } \\
\text { care services }\end{array}$ & 6.4 & 1 \\
$\begin{array}{l}\text { Foster a healthy and respectful } \\
\text { workforce environment }\end{array}$ & 5.9 & 2 \\
$\begin{array}{l}\text { Ensure efficient and accountable } \\
\text { leadership and governance }\end{array}$ & 4.9 & 3 \\
$\begin{array}{l}\text { Efficient use of hospital financing } \\
\text { Maximise innovations and the use of }\end{array}$ & 4.1 & 4 \\
health technologies & 2.7 & 5 \\
$\begin{array}{l}\text { Trigger health system audits } \\
\text { Initiate new healthcare reform }\end{array}$ & 2 & 6 \\
\hline
\end{tabular}

meeting quality-of-care needs. We identified high patients' wait time, biased patient-provider interactions, low treatment and care services, disrespect of patients, missing patient's medical records, patient's dissatisfaction, hazardous work area and incongruous infrastructure as the major challenges at the public hospitals. Reinforcing patientcentred quality-of-care services will be ensured at the level of public hospitals in Ethiopia as follows:

\section{'Whole-person' care}

The quality outcomes of public hospitals in Ethiopia can be improved through the coordination of health, behavioural health and social services in a patient-centred manner. This will help patients interact in the hospitals with coordinated system, receive truly integrated services through appropriate providers and have all the freedom, dignity and respect to all citizens.

Key interventions:

- Perform on-going assessment and reporting of health disparities in order to ensure well-integrated physical, behavioural and social health services

- Establish standards-of-care for persons with disabilities in order to develop an understanding of patients as an individual, not as a disease, and to ensure availability of appropriate services for people with disabilities

- Take account of the particular needs of patients within specific groups when planning and delivering care

- Modify the physical layout of public hospitals to provide access for people with mobility difficulties 
- Clearly identify unmet target population who receive the hospitals' services in order to identify priorities to reduce health inequalities, avail enough healthcare assistance and plan improvements for access and inclusion.

\section{Coordination and communication}

Implementing a holistic, ethically imperative, coordination and communication approach across public hospitals enhances effective exchange of information in a timely manner, improves transitions of care, reduces administrative overhead, and ensures patient-centred quality-of-care. Effective communication is required to effectively meet patients' expectations and to engage them actively to participate in the decision-making process of the hospitals.

Key interventions:

- Ensure accurate and complete coding of documentation in order to deliver all relevant information to patients whenever they move between services and to ensure that they are delivered the best possible care

- Plan to improve healthcare services in line with the best practices recommended by the Ethiopian National Accreditation Office and other international standards

- Seek regular feedback from patients about their experience of the hospitals' services to evaluate the impact of service interventions and ensure that the preferences and views of patients are taken into account

- Establish strong communication platforms between departments in the hospitals to optimise patient's flow

- Simplify exchange of patient medical records through well-secured electronic communication technologies

- Release information to the community about what healthcare services are available at the hospital and how people can get to them.

\section{Patient support and empowerment}

Public hospitals should provide patients with the opportunities and environment to develop the skills, confidence and knowledge to move from being a passive recipient of care to an active partner in their healthcare. Key interventions:

- Reshape morning patient health education sessions to be much more easily stimulating and understandable way that patients interact, ask questions and express needs and expectations

- Enable patients to understand their health condition, take responsibility for their health and actively seek care only when necessary by creating and providing them with a patient's information brochure.

\section{Ready access to healthcare}

Public hospitals need to provide ready access to care in the most advanced possible way. The public hospitals should offer healthcare services with more advanced technologies uninterruptedly. The FMoH medium-term HSTP shall advocate for a ready access to care at tertiary hospitals level.

Key interventions:

- Redesign advanced models of care for patients with emergency and urgent healthcare needs

- Provide ambulatory care for the community in a more advanced and well-prepared way

- Digitalise hospital discharge planning to make beds ready for access

- Establish a continuous quality improvement process to identify and address problems

- Establish a proper and integrated linkage between public hospitals for patient referral systems

- Improve and automate diagnostic services to enhance quality-of-care delivery.

\section{Autonomy}

Public hospitals should reinforce keeping the rights of patients to determine their medical care without their healthcare provider trying to influence the decision.

Key interventions:

- Protect the right of patients to be given information about their medical diagnosis, treatment and progress, except in the interest of protecting others who may be harmed by the patient's decisions.

- Keep patient's medical records and confidential information in a way it can be archived only by an allowable person.

\section{Strategic priority II: foster a healthy and respectful workforce environment \\ Rationale}

Job satisfaction of the health workforce in the public hospitals was low, with only $25 \%$ of job-satisfied workforce engaged. Lack of appropriate salary and benefits, continuing education, management style, tasks, work environment, workload, moral satisfaction and job stability were among the major causes. Strategic priority II shall be delivered at the level of public hospitals in the following way:

\section{Appropriate staffing}

Assigning a competent workforce that matches with patient load could decrease the likelihood of death or serious complications of patients and minimise the diagnosis, care and treatment errors.

Key interventions:

- Clarify the existing number of staff and their specific roles within case teams to ensure clear job descriptions are in place for all staff to understand and abide by

- Monitor staffing requirements to ensure adequate staff with appropriate competencies and attributes are in place as guided by the $\mathrm{FMoH}$

- Develop a robust workforce plan to ensure quality patient care

- Ensure skilled and passionate staff are used effectively to provide excellent healthcare services 
- Promote a safe and supportive working environment, free of harassment and discrimination, for all workers, regardless of their political view, gender, ethnicity, age or any other aspect of difference.

\section{Meaningful recognition}

Recognition of public health workforce to reward their work and behaviours is a fundamental need for them to extend their determinations towards meeting the intended goals of the hospitals. The recognition shall be made in such a way that it is relevant to the actual needs of staff.

Key interventions:

- Regularly monitor staff service performance data to reward and attract best-performing providers who bring innovative ideas and procedures

- Well-mentor, develop and train staff to promote professional development and to ensure that their expertise and skills are not lost in the organisation

- Promote outstanding employees through newsletters and forums to encourage providers to develop strong personal accountability and empowerment to achieve the hospital's goals

- Identify functions and activities that supervisor-level hospital staff could delegate to their lower level staff in order to make staff feel more valued and respected

- Expand the range of employee income through further provision of private-wing and other initiatives to compensate for salary dissatisfaction, which was identified in the current study as the major reason for job dissatisfaction

- Negotiate more with Ethiopian Housing Development Agency for staff housing to enable staff early benefit from the condominium housing strategy intended for government employees.

\section{Professional development}

Professional development opportunities for public hospital workforce shall be recognised as critical in maintaining a skilled public health workforce and ensure job satisfaction. The hospital management needs to strive for personal and professional growth of staff.

Key interventions:

- Regularly re-evaluate the skills of the workforce to identify and rectify any skills deficits or training requirements to use their full potential

- Prevent unfair and unjust differences among staff in opportunities for training and career progression

- Ensure all staff receive appropriate training regarding the hospital's mission, vision, values and goals

- Develop and implement a clearly defined career path for all health staff as indicated in the Continuing Professional Development (CPD) guideline for health professionals in Ethiopia ${ }^{45}$

- Design and implement an organisational development plan to ensure job satisfaction among staff and sense pride to work in the hospitals
- Ensure all staff are appropriately trained and are encouraged and supported to maintain their competence to help them grow and develop professionally

- Identify and implement different online, distance and face-to-face educational accesses as continuing professional development options for staff, and facilitate exchange visits for staff for information sharing.

\section{Skilled communication}

A culture of professional communication skills shall be ensured to create a common understanding of ideas, desires and observations between patients and providers, hospital management and providers, and among providers.

Key interventions:

- Provide timely, fair and accurate performance feedback

- Create an environment where individuals from diverse backgrounds feel included, celebrated and respected

- Emphasise professional and personal diversity as a competitive advantage which could promote core values and professional standards

- Strengthen communication throughout the hospitals to develop a culture that encourages innovation, collaboration and the free exchange of ideas

- Foster a positive and welcoming environment through nurturing a culture of respect, inclusion and equal opportunity.

\section{Strategic priority III: ensure efficient and accountable leadership and governance \\ Rationale}

There have been visible leadership gaps which contributed to poor implementation and deprived results of hospitals' services. Lower competence and commitment of top management to support the reform efforts, not involving technical staff in various decision-making, not organising job-related trainings to staff and lower management capacity in leading quality of services were among critical governance gaps. Strategic priority III should be delivered in the following way:

\section{Collaborative organisational culture}

Hospital managements should lead the values and behaviours of their staff towards a system of shared assumptions.

Key interventions:

- Strengthen systems to monitor and communicate decision-making and ensure consistent and timely communication and messaging for feedback, constructive dialogue and on-going strategy development

- Decentralise management with as much delegation of authority and responsibility as possible

- Adapt a modern and efficient management structure and systems which could create a safe, fair and stimulating working environment for all staff 
- Recruit and retain skilled and motivated hospital managers who could provide models of good practice in management and motivation of staff

- Developing an effective communication strategy which facilitates communication between staff and management at all levels

- Pursue good governance through capacity building of management teams.

\section{Partnership}

Public hospitals develop and strengthen partnership to advance their mutual interests to achieve their mission and amplify their extent.

Key interventions:

- Collaborate and build partnership with other public and private hospitals in targeted areas to meet common goals

- Collaborate with universities, research institutes and professional associations for mutual interests

- Expand affiliations and partnerships with development partners to create novel interdisciplinary and interprofessional programmes, approaches and projects that could ensure sustainability of the hospitals.

\section{Strategic priority IV: enhance efficiency of hospital financing \\ Rationale}

The healthcare financing constrained with availability of drugs, reagents and other needed supplies. Though there were efforts in increasing hospital revenues, financial mobilisation and budget consumption were weak. Besides, corruptions were shown to exist due to poor healthcare monitoring systems. Strategic priority IV could be delivered in the following way:

\section{Responsible stewardship}

The hospitals are required to exercise ethics for a responsible planning and management of resources. The hospitals' financial management system should ensure the capacity needed to manage resources effectively.

Key interventions:

- Ensure maximum efficiency in the distribution and use of all hospital resources

- Ensure state-of-the-art facilities and infrastructure

- Maximise and leverage philanthropic opportunities through training of the hospitals' finance and administration staff.

\section{Resource mobilisation}

Public hospitals need to maximise their sources of income from domestic and international resources. They should involve identifying other institutions that share the same values with them and take steps to manage the relationship.

Key interventions:

- Appropriately allocate resources and demonstrate value for revenues made
- Diversify revenue sources to establish a financially strong hospital which is responsive to changing financial conditions

- Seek a broad base of philanthropic support from communities and partners to involve in developing donor relationships to meet fundraising goals

- Design and implement infrastructure that supports in producing resources. This could be done by collaboration and agreement lease with private companies that supply equipment

- Provide sufficient resources including drugs, supplies and equipment to allow the hospitals to perform the required tasks.

\section{Strategic priority V: maximise innovations and the use of} health technologies

\section{Rationale}

The hospitals lack up-to-date and advanced technologies such as automated laboratory equipment, medical devices and IT. This forces the hospitals to refer their patients to private sector health facilities to get advanced diagnostic or therapeutic procedures, which could be unaffordable for patients. The network infrastructure of the hospitals to connect between departments or with other hospitals is also limited. The healthcare providers are not supported or motivated to conduct researches which could advance hospital services and ensure sustainability.

Key interventions:

- Implement operational researches to prioritise future growth and development of health services and ensure hospital sustainability

- Encourage knowledge sharing and collaboration between hospital staff and other researchers and among professionals in order to improve patient care

- Strengthen electronic medical recording system across hospital departments and computerise management of supplies and drugs

- Document, disseminate and continually update standard operating procedures of hospitals' services

- Assess needs and train staff on IT to ensure they have basic computer skills

- Train staff on preventive and curative maintenance procedures on basic medical equipment.

\section{DISCUSSION}

The Ethiopian healthcare reform implemented in the form of BPR had its most important goal to improve healthcare services. The Ethiopian government introduced the reform to divert the multiple challenges tracked from clients' symptoms and on-site evaluations, which witnessed a complicated structure across healthcare facilities. According to our findings, the reform was able to restructure the hospitals' departments into case teams, with the goal of adopting a 'one-stop shopping' approach. However, $50 \%$ of healthcare providers who have been working in the study sites from the inception 
of the reform described that the reform was not effective to satisfy the perceived health service needs. Limited effects were reported in favour of healthcare quality $(48 \%)$, access $(50 \%)$, efficiency $(51 \%)$, sustainability $(53 \%)$ and equity $(61 \%)$. While, poor effects were reported in patient-provider $(41 \%)$ and provider-management $(32 \%)$ interactions. The anticipated patientprovider interaction depicted in the reform document has not been achieved. A mutual construction and maintenance of trust between patient and provider would generate smooth interaction between the two parties, and the presence of this trust further insists the community decide to engage in prevention and control interventions in the general healthcare systems. ${ }^{46}$ Though the reform fairly improves turn-around time of hospital services as reported by a relatively high number of respondents, a higher rate of patient appointment time, longer waiting time on reception, low patient satisfaction, and poor treatment and respect given to patients by providers continued to challenge the system. Recent studies conducted in Ethiopia ${ }^{14}{ }^{16}$ also came up with similar findings. We emanated strategic priority I, 'reinforce patient-centred quality of care services', to address the identified gaps that highly compromised patient care.

In healthcare sectors that need to undergo healthcare reform, availability of resources and competency of the workforce are essential but not sufficient to ensure the desired worker performance. Rather, effectiveness of the reform is critically dependent on workforce job satisfaction and motivation. For this reason, healthcare reform efforts need to focus on job and tasks becoming more conducive to patients and work to improve staff job satisfaction. Our findings indicate that the reform did not come up with encouraging results regarding the attention given to the health workforce. These findings also concur with previous studies held in Ethiopia. ${ }^{47-49}$ Poor provider-management interaction in public hospitals would compromise the maximum commitment and engagement providers could exert to their duties. This in turn leads the hospitals not to function to the best of their abilities. If there are factors which are beyond the control of providers, the possibilities that they become client oriented, productive, and skilled and competent to perform their duties would lessen. ${ }^{26}$ While hospitals with higher management practice scores had better clinical outcomes, higher levels of patient satisfaction and better financial management. ${ }^{50}$ We proposed strategic priority II, 'foster a healthy and respectful workforce environment', to tackle the identified health workforce gaps.

The analysis revealed that the commitment and support of top managements towards the reform was not agreeable $(61 \%)$. Senior leadership is a necessary prerequisite for successful re-engineering, not just any senior manager will do since leaders can literally 'make or break' a BPR project. ${ }^{21}$ Strengthening the capacity of the top managements themselves is important for them to show up competently and deliver their level best, and ensure that reform implementing staff are convinced by the intended reform. Staff may fear losing jobs, benefits and status, and these cause staff resistance to the BPR. ${ }^{3}$ Strong leadership style should be in place to create an environment where employees affected by BPR project understand its objective and are involved throughout the process. ${ }^{38}$ The Ethiopian public hospital staff must understand the overall function of core business processes in the hospitals and internalise it. Similarly, business process owners who actually are responsible for the hospitals' various operational functions need to monitor the reengineering day-to-day efforts. The management's support and involvement in all phases of the BPR reform efforts is essential for the success of BPR implementation ${ }^{15}$ We proposed strategic priority III, 'ensure efficient and accountable leadership and governance', from the leadership and governance gaps we identified.

Financial management systems raised another key concern in the re-engineered public hospitals. The reform was able to slightly increase income of the hospitals. However, the hospitals' healthcare financing system remained weak: financial mobilisation schemes did not centre on evidence-based plans and their budget consumption system was stagnant. The hospitals are suspected of corruption, and this could contribute to the financial management gaps observed. The different financial management problems mentioned could justify that the BPR healthcare reform implemented in the hospitals was far behind the target of economic dimension of healthcare access. The finding of this study was in support of other studies held in Ethiopia. ${ }^{13} 14$ The FMoH also verified in its Health Sector Development Program (HSDP) IV document that health financing remains a major challenge in the health system of Ethiopia. ${ }^{51}$ In an attempt to be self-reliant in financing the provision of essential health services, Ethiopia should resolve to make its own investments in the health of its people. ${ }^{52}$ However, the positive financial interventions the hospitals were undertaking are opening room for improvement. For instance, there were positive trends that incomes of the hospitals are increasing and the required financial resources are emerging. We proposed strategic priority IV, 'enhance efficiency of hospital financing', based on the findings of the study which identified financial resources as the major influencing factor of the reform.

It was reported that Ethiopian public hospitals are challenged by the lack, scarcity and inappropriate usage of medical products and technologies. ${ }^{44}$ The current study also revealed the same. There were scarcities of drugs, medical supplies, medical apparatus and equipment in the hospitals. The movement towards initiating new practices for effective and advanced usage of technologies was poor. Lacking to exercise appropriate information communication and exchange methodologies were also realised. The reporting systems of the hospitals were shown to be complex and time consuming. The monitoring and evaluation system already in place was 
weak to appropriately capture, analyse and disseminate information relevant to staff. The hospitals have been highly affected by the lack of sufficient internet access in the hospitals, which could deactivate staff from updating their knowledge and translate to their patients. IT could offer significant opportunities for patient-physician concordance, ${ }^{53}$ improve patient information services ${ }^{54} 55$ and offer clinicians with quick and easy access to information. ${ }^{56} \mathrm{We}$ propose strategic priority $\mathrm{V}$, 'maximize innovations and the use of health technologies', to mitigate the gaps in availability and usage of IT.

This study has some limitations. The study did not solicit the views of administrative staff and we think their inputs may have further strengthened the study findings, while the possible effect of this has been mitigated by inclusion of healthcare providers who also have administrative role. The study bases the perspectives of the respondents that may increase the likelihood of recall biases. There was no control site in the study to validate the observations in the study sites or to rule out possible confounders. However, the respondents included were those who were hired before the inception of the reform to select respondents who knew the performances of the study sites before implementation of the reform and who could better analyse the changes that occurred due to the reform.

\section{CONCLUSION}

In light of the weak healthcare system in resourcelimited countries, such as Ethiopia, it is necessary to seek for strategies and policies that could support health service capacity at the ground level. Equally, effective operational, clinical and governance functions of health systems, as well as a motivated and committed health workforce, are important to move healthcare reform processes forward. Political commitments at this junction might be critical, though there needs to be a clear demarcation between political and technical engagements. We provide evidence that the BPR healthcare reform implemented in Ethiopia seems unable to improve access, equity, quality, efficiency and sustainability of healthcare services due to bootlicks in financial resources, top management commitment and support, working environment and IT. Disrupting all these status quos were required strategic priorities that are capable of strengthening healthcare reforms in such settings. Based on findings of phase I of the study, 5 strategic priorities, with 14 operational objectives and 67 potential interventions, which were likely to strengthen implementation of healthcare reform are proposed based on their strategic priority, which were: reinforce patient-centred quality of care services; foster a healthy and respectful workforce environment; efficient and accountable leadership and governance; efficient use of hospital financing; and maximise innovations and the use of health technologies. The proposed strategies could be tested in some sites over a period and used to enrich the quality of healthcare interventions through continuous review, refinement and adjustment of the reform as required. We recommend future researches be conducted in other resource-limited countries.

Acknowledgements The authors would like to thank healthcare providers and senior health policy experts in Ethiopia for their time and kindness to participate in the study.

Contributors TM conceived the study. TM, MCM and MJO designed the study. TM implemented the study under the guidance of MCM and MJO. TM wrote the first draft, and MCM and MJO critically revised it for important intellectual content. TM and MCM analysed and interpreted data. All authors participated in the write-up, review, feedback and approval of the manuscript for publication.

Funding This research project has been supported by the University of South Africa.

\section{Competing interests None declared.}

Ethics approval Higher Degrees Committee of the Department of Health Studies, University of South Africa and (UNISA); and the Research and Technology Transfer Core-process of the Addis Ababa City Administration Health Bureau.

Provenance and peer review Not commissioned; externally peer reviewed.

Data sharing statement No additional data are available.

Open Access This is an Open Access article distributed in accordance with the Creative Commons Attribution Non Commercial (CC BY-NC 4.0) license, which permits others to distribute, remix, adapt, build upon this work noncommercially, and license their derivative works on different terms, provided the original work is properly cited and the use is non-commercial. See: http:// creativecommons.org/licenses/by-nc/4.0/

\section{REFERENCES}

1. Yasin M, Augusto M, Lisboa J, et al. Assessing the competitive effectiveness of hospitals: the role of quality improvement initiatives. Total Qual Manag Bus Excell 2011;22:433-42.

2. Vogel JP, Oxman AD, Glenton C, et al. Policymakers' and other stakeholders' perceptions of key considerations for health system decisions and the presentation of evidence to inform those considerations: an international survey. Health Res Policy Syst 2013;11:19.

3. Debela T. Business process reengineering in Ethiopian public organizations: the relationship between theory and practice. J Bus Adm Stud 2009;1:20-59.

4. Fox AM, Reich MR. The politics of universal health coverage in lowand middle-income countries: a framework for evaluation and action. $J$ Health Polit Policy Law 2015;40:1023-60.

5. Gómez EJ, Ruger JP. The global and domestic politics of health policy in emerging nations. J Health Polit Policy Law 2015;40:3-11.

6. Yasin MM, Gomes CF, Miller PE. Competitive strategic grouping for hospitals. Total Qual Manag J 2011;23:301-2.

7. Austin WJ. The incommensurability of nursing as a practice and the customer service model: an evolutionary threat to the discipline. Nurs Philos 2011;12:158-66.

8. Bertolini M, Bevilacqua M, Ciarapica FE, et al. Business process re-engineering in healthcare management: a case study. Bus Process Manag J 2011;17:42-66.

9. Caccia-Bava MC, Guimaraes VCK, Guimaraes T. Important factors for success in hospital BPR project phases. Int J Health Care Qual Assur 2013;26:729-45.

10. McNatt Z, Thompson JW, Mengistu A, et al. Implementation of hospital governing boards: views from the field. BMC Health Serv Res 2014:14:178.

11. Menberu AW. The ups and downs of Business Process Re-engineering (BPR): a tale of two offices in Bahir Dar town, Ethiopia. 1st Annual International Interdisciplinary Conference Proceedings; 24-26 April 2013. Azores, Portugal.

12. Jamali $\mathrm{G}$, Abbaszadeh MA, Ebrahimi M, et al. Business process reengineering implementation: developing a causal model of critical success factors. Int $J$ e-Educ e-Bus e-Manag e-Learn 2011;1:354-9.

13. Bradley $\mathrm{EH}$, Byam $\mathrm{P}$, Alpern $\mathrm{R}$, et al. A systems approach to improving rural care in Ethiopia. PLOS ONE 2012;7:e35042. 
14. Beyene W, Jira C, Sudhakar M. Assessment of quality of health care in Jimma zone, southwest Ethiopia. Ethiop J Health Sci 2011;21:49-58.

15. Saad GH. Empirically-based rules and extensions for improving business process reengineering practice. APUBEF proceedings 2010:1-8

16. Maleki $\mathrm{T}$, Beikkhakhian $\mathrm{Y}$. Business process reengineering implementation: an investigation of critical success factors. International Conference on Information and Finance, IPEDR 21; 2011, Singapore.

17. McHugh M, Dyke KV, McClelland M, et al. Improving patient flow and reducing emergency department crowding: a guide for hospitals. Rockville, MD: Agency for Healthcare Research and Quality, 2011.

18. Alhassan RK, Spieker N, van Ostenberg P, et al. Association between health worker motivation and healthcare quality efforts in Ghana. Hum Resour Health 2013;11:37.

19. Ramanigopal CS, Palaniappan G, Hemalatha N, et al. Business process reengineering and its applications. Int J Manag Res Rev 2011;1:275-88

20. Mlay SV, Zlotnikova I, Watundu S. A quantitative analysis of business process reengineering and organizational resistance: the case of Uganda. Afr J Inf Syst 2013;5:1

21. Goksoy A, Ozsoy B, Vayvay O. Business process reengineering strategic tool for managing organizational change an application in a multinational company. Int J Bus Manag 2012;7:89-112.

22. Habib MN, Wazir MI. Role of education and training in the successful implementation of business process reengineering: a case of public sector of Khyber PakhtunKhwa (KPK). World J Soc Sci 2012;2:172-85.

23. Srikanth A. Significance of BPR \& ERP implementation in healthcare industry: an exploratory research. Int J Manag Strateg 2012;3:5.

24. Senyonjo M. The African Regional Human Rights System. 30 years after the African charter on human and peoples' rights. Leiden, the Netherlands: Koninklijke Brill NV, 2012.

25. Chuma J, Okungu V. Viewing the Kenyan health system through an equity lens: implications for universal coverage. Int $J$ Equity Health 2011;10:22

26. Lutwama GW, Roos JH, Dolamo BL. A descriptive study on health workforce performance after decentralisation of health services in Uganda. Hum Resour Health 2012;10:41.

27. Okuonzi PV. Learning from failed health reform in Uganda. BMJ 2007;329:1173-6.

28. Flessa S, Moeller M, Ensor T, et al. Basing care reforms on evidence: the Kenya health sector costing model. BMC Health Serv Res 2011;11:128.

29. Frumence $\mathrm{G}$, Nyamhanga T, Mwangu M, et al. Challenges to the implementation of health sector decentralization in Tanzania: experiences from Kongwa district council. Glob Health Action 2013;6:20983.

30. Doherty JE. Regulating the for-profit private health sector: lessons from East and Southern Africa. Health Policy Plan 2015;30(Suppl 1):93-102.

31. Monette DR, Sullivan TJ, DeJong CR. Applied social research: a tool for the human services, 8th edn. Belmont, USA: Brooks/Cole, Cengage Learning, 2011.

32. Knowles JC, Leighton C, Stinson W. Measuring results of health sector reform for system performance: a handbook of indicators. Special Initiatives Report No. 1. Bethsaida, MD: Partnerships for Health Reform, Abt Associates, 1997.

33. Donabedian A. Evaluating the quality of medical care. Milbank $Q$ 2005;83:691-729.

34. Adds Ababa City Administration Health Bureau (AAHB). Standard operation procedure for the delivery of medical services. Addis Ababa, Ethiopia: AAHB curative services core process, 2008.
35. Fortney JC, Burgess JF, Bosworth $\mathrm{HB}$, et al. A re-conceptualization of access for 21st century healthcare. J Gen Intern Med 2011;26 (Suppl 2):639-47.

36. Comber AJ, Brunsdon C, Radburn R. A spatial analysis of variations in health access: linking geography, socio-economic status and access perceptions. Int J Health Geogr 2011;10:44.

37. Levesque JF, Harris MF, Russell G. Patient-centred access to health care: conceptualising access at the interface of health systems and populations. Int J Equity Health 2013;12:18.

38. Aghdasi M, Albadvi A, Ostadi B. Desired organisational capabilities (DOCs): mapping in BPR context. Int $J$ Prod Res 2010;48:2029-53.

39. Hsu C, Sandford BA. The Delphi Technique: making sense of consensus. Pract Assess Res Eval 2007;12:10.

40. Mora M. Research methodologies, innovations and philosophies in software systems engineering and information systems. Hershey, USA: IGI Global, 2012.

41. Yager RR, Kacprzyk J. The ordered weighted averaging operators: theory and applications. Springer, USA: Springer Science \& Business Media, LLC, 2012.

42. Black K. Business statistics: for contemporary decision making, 7th edn. New Jersey, USA: John Wiley \& Sons, 2011

43. Vaus DD. Surveys in Social Research: social research today. London, UK: Routledge, 2013.

44. Federal Democratic Republic of Ethiopia Ministry of Health (FMoH). Health Sector Transformation Plan (HSTP): 2015/16-2019/20. Addis Ababa, Ethiopia: FMoH, 2015.

45. Ethiopian Food, Medicine and Healthcare Administration and Contro Authority. Continuing Professional Development [CPD] guideline for health professionals in Ethiopia. Addis Ababa, Ethiopia: Federal Democratic Republic of Ethiopia Ministry of Health, 2013.

46. Simonds VW, Christopher S, Sequist TD, et al. Exploring patient-provider interactions in a Native American community. $J$ Health Care Poor Underserved 2011;22:836-52.

47. Negussie N, Costantinos BT. An analysis of factors affecting performance of nurses in public hospitals and health centers in Addis Ababa [MA thesis]. Addis Ababa, Ethiopia: Addis Ababa University, 2010.

48. Negussie N. Relationship between rewards and nurses' work motivation in Addis Ababa hospitals. Ethiop $\mathrm{J}$ Health $\mathrm{Sci}$ 2012;22:107-12.

49. Ethiopian Midwifes association (EMA). The state of the Ethiopia's Midwifery 2012: based on Ethiopian midwives association database. Addis Ababa, Ethiopia: Ethiopian Midwifes association and United Nations Population Fund, 2012.

50. Dorgan S, Layton D, Bloom N, et al. Management in Healthcare: why good practice really matters. New York, USA: McKinsey \& Company, 2013.

51. Federal Democratic Republic of Ethiopia Ministry of Health (FMoH). Health Sector Development Program IV, 2010/11-2014/14. Addis Ababa, Ethiopia: FMOH, 2010.

52. HaileMariam D. Prospects for self-reliance in the provision of public health services. Ethiop J Health Dev 2010;24:1.

53. Laugesen J, Hassanein K, Yuan Y. The impact of internet health information on patient compliance: a research model and an empirical study. J Med Internet Res 2015;17:e143.

54. Bujnowska-Fedak MM. Trends in the use of the Internet for health purposes in Poland. BMC Public Health 2015;15:194.

55. Callen J, Giardina TD, Singh H, et al. Emergency physicians' views of direct notification of laboratory and radiology results to patients using the internet: a multisite survey. J Med Internet Res 2015;17:e60.

56. Papi A, Ghazavi R, Moradi S. Determining the level of awareness of the physicians in using the variety of electronic information resources and the effecting factors. J Educ Health Promot $2015 ; 4: 49$. 